\title{
Spontaneous regression of hepatocellular carcinoma in a Caucasian male patient: A case report and review of the literature
}

\author{
DANIEL P. NOIJ and PETRUS W.G. VAN DER LINDEN \\ Department of Internal Medicine, Spaarne Gasthuis, 2035 RC Haarlem, The Netherlands
}

Received May 4, 2016; Accepted July 15, 2016

DOI: $10.3892 / \mathrm{mco} .2016 .1115$

\begin{abstract}
Hepatocellular carcinoma (HCC) is the most common primary hepatic cancer. As it often presents at an advanced stage, it generally has a poor prognosis. Spontaneous regression is a rare finding in HCC, and is often associated with tumour ischemia or a systemic inflammatory response. We herein present the case of a Caucasian patient with spontaneous regression of advanced HCC, not associated with either mechanism. Our patient was a 74-year old Caucasian male who presented with advanced biopsy-proven HCC, with $\alpha$-fetoprotein levels of $>16,600 \mathrm{kU} / 1$. On computed tomography examination, multiple metastases were identified. Due to the poor performance status and as the patient declined treatment, he was referred to a general practitioner for supportive palliative care. Six months later, the patient was alive and feeling well. He had suffered a cerebrovascular accident with ensuing partial hemiparesis, and had been initiated on enalapril, furosemide and curcumin. On re-evaluation, the lung lesions had disappeared, whereas both the liver and peritoneal lesions had decreased in size. Following a review of the literature, the conclusion was that this case of spontaneous regression of advanced HCC was not caused by ischemia, inflammation, or any other previously reported mechanism. Further research is required to elucidate the mechanisms underlying spontaneous regression of $\mathrm{HCC}$.
\end{abstract}

\section{Introduction}

Primary liver cancer is the seventh deadliest type of cancer, with a 5-year survival rate of $18 \%$. The annual increase in the incidence of primary liver cancer indicates that this is among the most rapidly increasing cancer incidences $3.7 \%$ in men and $3.0 \%$ in women) (1). Over $90 \%$ of primary liver cancer

Correspondence to: Dr Petrus W.G. van der Linden, Department of Internal Medicine, Spaarne Gasthuis, Boerhaavelaan 22, 2035 RC Haarlem, The Netherlands

E-mail: pw.vdlinden@kg.nl

Key words: case report, hepatocellular carcinoma, spontaneous regression, European Continental Ancestry Group, curcumin cases are hepatocellular carcinomas (HCCs). The majority of the patients present with advanced-stage disease, with only palliative treatment options available, including sorafenib and transarterial chemoembolization (2). Sorafenib is a multikinase inhibitor that is used as first-line palliative treatment in patients with advanced HCC; it improves the overall survival (mean, 2.8 months) and is reasonably well-tolerated, with the main side effects being diarrhoea, weight loss and hand-foot skin reaction (3).

Several cases of spontaneous tumour regression in HCC have been reported. The estimated incidence of spontaneous regression is $0.4 \%$ in HCC patients (4). Different hypotheses have been suggested, including tumour ischemia (5), systemic inflammatory reactions, discontinuation of immunosuppressive therapy (6), abstinence from alcohol consumption or the use of herbal preparations.

We herein report a case of regression of untreated metastasized HCC that not associated with any of the abovementioned mechanisms.

\section{Case report}

A 74-year old Caucasian male patient was admitted to our hospital with a 6-week history of malaise, loss of appetite, increased abdominal circumference, epigastric tenderness and a $20-\mathrm{kg}$ weight loss. The liver was non-tender and palpable $5 \mathrm{~cm}$ below the costal margin. A firm, non-tender mass, $2 \mathrm{~cm}$ in diameter, was detected in the epigastric angle. There was no lymphadenopathy or a rectal mass.

The patient's medical history included hepatic steatosis, hypertension, diabetes mellitus type 2 and percutaneous transluminal coronary angioplasties after a myocardial infarction. He had been prescribed insulin, metformin, pantoprazole, isosorbide mononitrate, enalapril, clopidogrel, felodipine, simvastatin, temazepam and metoprolol. The patient was a non-smoker and consumed 2 units of alcohol daily.

Hepatitis B and C and human immunodeficiency virus serology were assessed with the enzyme immunoassay method and were negative.

A computed tomography (CT) scan revealed multiple liver and lung lesions suspicious for metastases, peritoneal depositions, but no primary tumour (Fig. 1). An ultrasound-guided liver biopsy was performed. The biopsy revealed malignant cells positive for pancytokeratin, slightly positive for cytokeratin (CK) 7, $\alpha$-fetoprotein (AFP), carbohydrate 

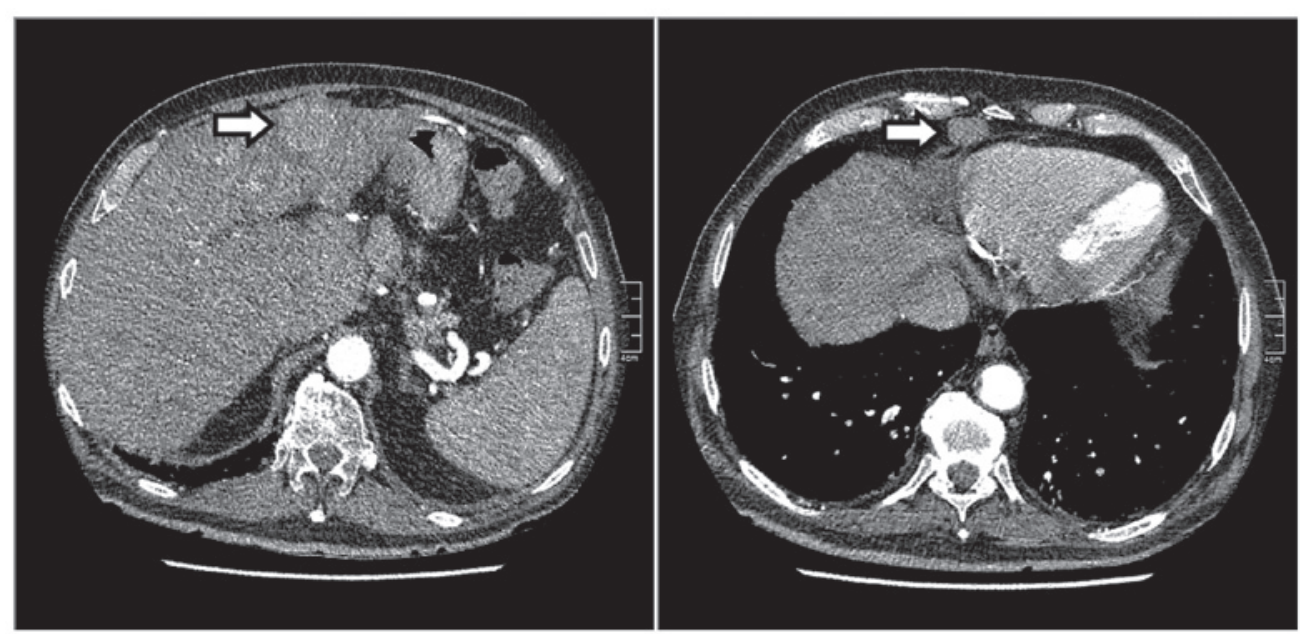

Figure 1. Initial computed tomography images showing a suspicious hepatic lesion and a lesion appearing to be malignant anterior to the pericardium (arrows).
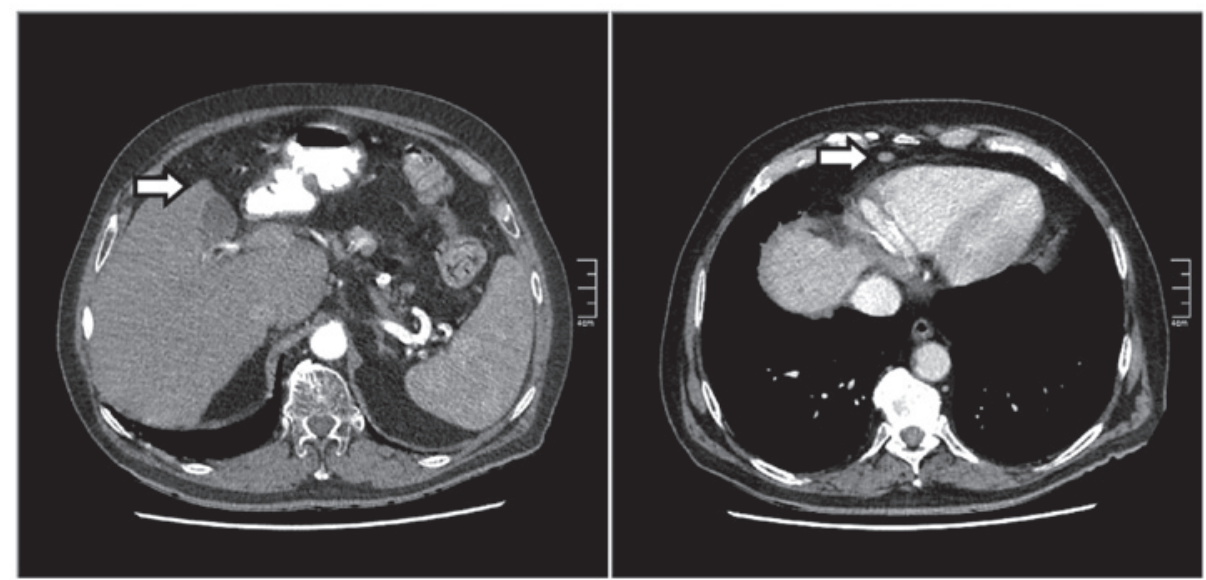

Figure 2. Follow-up computed tomography images at 6 months after initial diagnosis. The liver had significantly decreased in size. The suspicious hepatic lesion and the lesion anterior to the pericardium had also significantly decreased in size (arrows).

antigen-125 and CD-10, and negative for CK20, CDX-2, thyroid transcription factor-1, prostate-specific antigen, CK7 and monoclonal carcinoembryonic antigen (CEA), findings consistent with an undifferentiated carcinoma. Additional immunostaining was positive for hepatocyte paraffin 1 monoclonal antibody, and polyclonal CEA canalicular immunostaining was also present. Combined with a serum AFP level of $>16,600 \mathrm{kU} / \mathrm{l}$, the diagnosis of advanced HCC was established. Other laboratory tests are summarised in Table I. The patient had a poor performance status (WHO performance status 3) and declined any form of treatment. Therefore, he was referred to the general practitioner for supportive palliative care.

Six months later, the patient attended the outpatient clinic for re-evaluation of his disease. Over that time he had suffered a cerebrovascular accident (CVA) with ensuing partial hemiparesis. The AFP level had decreased to $1,795 \mathrm{kU} / \mathrm{l}$. The CT scan revealed that the pulmonary lesion had disappeared, whereas the suspicious hepatic lesion and the lesion anterior to the pericardium had significantly decreased in size (Fig. 2). An ultrasound-guided biopsy was repeated and the pathological examination indicated the previously diagnosed HCC. An independent academic pathology department

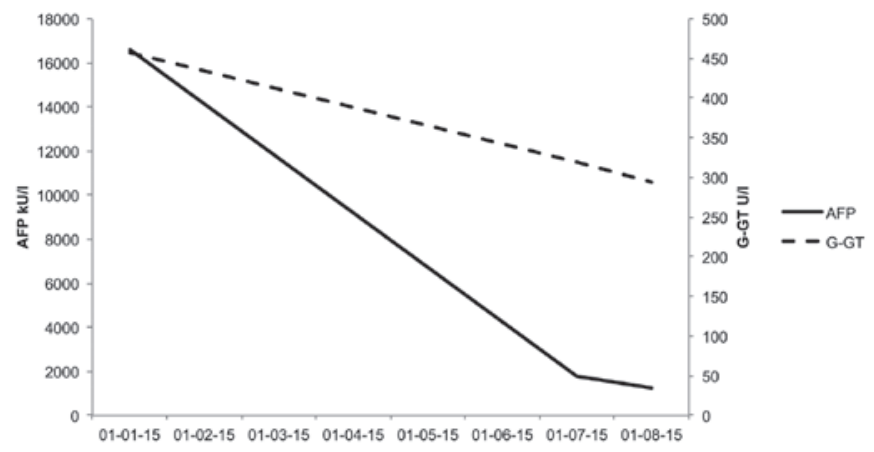

Figure 3. Decrease in serum $\alpha$-fetoprotein (AFP) levels and gamma-glutamyl transferase (G-GT) levels over time.

confirmed the results. The patient had gained $4 \mathrm{~kg}$ in weight over 6 months and maintained the consumption of 2 units of alcohol per day; he also reported improvement of his overall condition on a weekly basis. Two months after the CVA, he had been initiated on enalapril, furosemide and cucurmin, whereas he discontinued the use of simvastatin due to the side effects. The patient had not used androgens or herbal preparations and did not suffer from any type of 
inflammatory disease. One month later, the AFP level further decreased to $1,252 \mathrm{kU} / 1$ (Fig. 3).

\section{Discussion}

The present case differs from other case reports on spontaneous regression of $\mathrm{HCC}$, as neither invasive diagnostic procedures (e.g., hepatic arteriography) nor any form of treatment was performed, and no apparent inflammatory reaction occurred. During follow-up, the patient suffered a CVA and started using enalapril, furosemide and curcumin, which require further evaluation.

Curcumin is a substance found in turmeric roots and is a ginger-like herb. Curcumin has shown antitumour properties in cell lines by blocking nuclear factor- $\kappa \mathrm{B}$, thereby inducing cell death in curcumin-sensitive cells (7). Moreover, curcumin may act as an iron chelator and as a hepcidin suppressor. Iron overload in hemochromatosis is associated with an increased risk of HCC (8). However, there are insufficient data to propose iron depletion for the treatment of HCC. The patient had used curcumin for only 2 months. Given the extent of the tumour regression, it is unlikely that this may be attributed to the use of curcumin for 2 months. Moreover, the optimal dosing for the use of curcumin in oncology is unknown. More research is required to assess a potential role for curcumin in cancer treatment.

The only new medications the patient received were furosemide and enalapril. To the best of our knowledge, furosemide has no therapeutic properties in cancer, although the combined use of angiotensin-converting enzyme inhibitors and curcumin has been associated with inhibition of angiogenesis and diminished tumour growth in HCC in mice (9).

Some of the other medications used by the patient have been associated with a decreased risk of HCC. However, these are unlikely causes of tumour regression, as the tumour developed while the patient was receiving these preparations. Aspirin, but not non-aspirin non-steroidal anti-inflammatory drugs (NSAIDs), has been associated with a reduced risk of HCC (risk ratio=0.59) in a large prospective observational study (10). The non-randomized allocation of treatment increases the risk of confounding of results. Our patient used clopidogrel, which is a non-aspirin NSAID. Metoprolol has been associated with an inhibition of low-density lipoprotein cholesterol synthesis in a study on human cell lines, thereby reducing the risk of HCC (11). Metformin has been associated with a decreased risk of $\mathrm{HCC}$ in a meta-analysis of 8 observational studies (odds ratio $=0.50$ ), whereas insulin usage increased the risk on HCC (odds ratio=1.62) (12). Metformin decreases insulin resistance and inhibits the mammalian target of rapamycin pathway, thereby inhibiting cell proliferation and inducing apoptosis (13). Simvastatin was associated with a decreased risk of HCC in a large epidemiological study (14); thus, its discontinuation would increase rather than reduce the risk of HCC. To the best of our knowledge, pantoprazole, felodipine and temazepam have no therapeutic properties in cancer.

Ischemia may have contributed to tumour regression, given the patient's history of cardiovascular and cerebrovascular disease. However, although the CT scan revealed calcifications of the superior mesenteric artery, there was no thrombosis of
Table I. Overview of laboratory tests.

\begin{tabular}{lccc}
\hline & & Admission & Six months \\
Parameters & Units & $1-2015$ & $7-2015$ \\
\hline Hb & $\mathrm{mmol} / 1$ & $8.4^{\mathrm{b}}$ & 9.4 \\
MCV & $\mathrm{fl}$ & 82 & 88 \\
Thrombocytes & $\mathrm{x} 10^{9} / 1$ & 242 & 194 \\
Leukocytes & $\mathrm{x} 10^{9} / 1$ & 7.7 & 9.7 \\
APTT & $\mathrm{sec}$ & 28 & - \\
PT & $\mathrm{sec}$ & 12.6 & - \\
Sodium & $\mathrm{mmol} / 1$ & 137 & 139 \\
Potassium & $\mathrm{mmol} / 1$ & 5.0 & 4.2 \\
Creatinin & $\mu \mathrm{mol} / 1$ & 74 & 75 \\
MDRD clearance & $\mathrm{ml} / \mathrm{min} / 1.73 \mathrm{~m}{ }^{2}$ & 90 & 89 \\
Total bilirubin & $\mu \mathrm{mol} / 1$ & 15 & 13 \\
AP & $\mathrm{U} / 1$ & $258^{\mathrm{a}}$ & 118 \\
G-GT & $\mathrm{U} / 1$ & $469^{\mathrm{a}}$ & $294^{\mathrm{a}}$ \\
AST & $\mathrm{U} / 1$ & $37^{\mathrm{a}}$ & 23 \\
ALT & $\mathrm{U} / 1$ & 24 & 33 \\
LDH & $\mathrm{U} / 1$ & $325^{\mathrm{a}}$ & $254^{\mathrm{a}}$ \\
Albumin & $\mathrm{g} / \mathrm{l}$ & 36.8 & 37.1 \\
Total protein & $\mathrm{g} / \mathrm{l}$ & 65.2 & 68.5 \\
Calcium & $\mathrm{mmol} / 1$ & 2.32 & 2.34 \\
AFP & $\mathrm{kU} / 1$ & $>16600.0^{\mathrm{a}}$ & $1794.7^{\mathrm{a}}$ \\
CEA & $\mathrm{ug} / \mathrm{l}$ & 1.2 & 1.1 \\
CA-15.3 & $\mathrm{kU} / 1$ & 14.6 & - \\
CA-19.9 & $\mathrm{kU} / 1$ & 11 & 18 \\
PSA & $\mathrm{ug} / 1$ & 1.1 & 0.90 \\
hCG & $<2.0$ & $<2.0$ \\
\hline & & & \\
\hline
\end{tabular}

${ }^{a}$ High value. ${ }^{b}$ Low value. Hb, haemoglobin; MCV, mean corpuscular volume; APTT, activated partial thromboplastin time; PT, prothrombin time; MDRD, Modification of Diet in Renal Disease; AP, alkaline phosphatase; G-GT, gamma-glutamyl transferase; AST, aspartate aminotransferase; ALT, alanine aminotransferase; LDH, lactate dehydrogenase; AFP, $\alpha$-fetoprotein; CEA, carcinoembryonic antigen; CA, carbohydrate antigen; PSA, prostatespecific antigen; hCG, human chorionic gonadotropin.

the hepatic artery or the portal vein. Moreover, the extrahepatic lesions decreased in size. Thus, there appears to be no possible route through which CVA exerted any effect on the regression of the HCC and its metastases. Thus, ischemia appears to be an unlikely cause of tumour regression in this patient.

Regression of HCC has been associated with systemic inflammatory responses (e.g., regression following cholangitis or capsular rupture) (5). It is unlikely that any inflammatory reaction occurred, given the absence of fever, leukocytosis, or elevation of the $\mathrm{C}$-reactive protein levels. It is therefore unlikely that inflammation contributed to tumour regression. More research is required to elucidate the mechanisms underlying the spontaneous regression of $\mathrm{HCC}$ in the present case.

In conclusion, we herein report a case of spontaneous regression of advanced HCC in a Caucasian male patient that may not be attributed to ischemia or inflammation. 


\section{Acknowledgements}

We would like to thank Professor JPH Drenth for his comments during the preparation of the manuscript.

\section{References}

1. Siegel RL, Miller KD and Jemal A: Cancer statistics, 2016. CA Cancer J Clin 66: 7-30, 2016.

2. Bruix J and Sherman M; American Association for the Study of Liver Diseases: Management of hepatocellular carcinoma: An update. Hepatology 53: 1020-1022, 2011.

3. Llovet JM, Ricci S, Mazzaferro V, Hilgard P, Gane E, Blanc JF, de Oliveira AC, Santoro A, Raoul JL, Forner A, et al: Sorafenib in advanced hepatocellular carcinoma. N Engl J Med 359: 378-390, 2008.

4. Oquiñena S, Guillen-Grima F, Iñarrairaegui M, Zozaya JM and Sangro B: Spontaneous regression of hepatocellular carcinoma: A systematic review. Eur J Gastroenterol Hepatol 21: 254-257, 2009.

5. Huz JI, Melis M and Sarpel U: Spontaneous regression of hepatocellular carcinoma is most often associated with tumour hypoxia or a systemic inflammatory response. HPB (Oxford) 14: 500-505, 2012

6. Kumar A and Le DT: Hepatocellular carcinoma regression after cessation of immunosuppressive therapy. J Clin Oncol 34: e90-e92, 2016.

7. Marquardt JU, Gomez-Quiroz L, Arreguin Camacho LO, Pinna F, Lee YH, Kitade M, Domínguez MP, Castven D, Breuhahn K, Conner EA, et al: Curcumin effectively inhibits oncogenic NF- $\mathrm{kB}$ signaling and restrains stemness features in liver cancer. J Hepatol 63: 661-669, 2015.
8. Kowdley KV: Iron, hemochromatosis, and hepatocellular carcinoma. Gastroenterology 127 (5 Suppl 1): S79-S86, 2004.

9. Nasr M, Selima E, Hamed O and Kazem A: Targeting different angiogenic pathways with combination of curcumin, leflunomide and perindopril inhibits diethylnitrosamine-induced hepatocellular carcinoma in mice. Eur J Pharmacol 723: 267-275, 2014

10. Sahasrabuddhe VV, Gunja MZ, Graubard BI, Trabert B, Schwartz LM, Park Y, Hollenbeck AR, Freedman ND and McGlynn KA: Nonsteroidal anti-inflammatory drug use, chronic liver disease, and hepatocellular carcinoma. J Natl Cancer Inst 104: 1808-1814, 2012.

11. Naegele H, Behnke B, Gebhardt A and Strohbeck M: Effects of antihypertensive drugs on cholesterol metabolism of human mononuclear leukocytes and hepatoma cells. Clin Biochem 31: 37-45, 1998.

12. Singh S, Singh PP, Singh AG, Murad MH and Sanchez W: Anti-diabetic medications and the risk of hepatocellular cancer: A systematic review and meta-analysis. Am J Gastroenterol 108: 881-891; quiz 892, 2013.

13. Jalving M, Gietema JA, Lefrandt JD, de Jong S, Reyners AK, Gans RO and de Vries EG: Metformin: Taking away the candy for cancer? Eur J Cancer 46: 2369-2380, 2010.

14. Lai SW, Liao KF, Lai HC, Muo CH, Sung FC and Chen PC: Statin use and risk of hepatocellular carcinoma. Eur J Epidemiol 28: 485-492, 2013. 